\title{
ORDERED COMPACTIFICATIONS AND FAMILIES OF MAPS
}

\author{
D. M. LIU and D. C. KENT \\ Department of Pure and Applied Mathematics \\ Washington State University \\ Pullman, WA 99163-3113, U.S.A.
}

(Received April 28, 1995 and in revised form June 2, 1995)

\begin{abstract}
For a $T_{3.5}$-ordered space, certain families of maps are designated as "defining families." For each such defining family we construct the smallest $T_{2}$-ordered compactification such that each member of the family can be extended to the compactification space. Each defining family also generates a quasi-uniformity on the space whose bicompletion produces the same $T_{2}$-ordered 'compactification.
\end{abstract}

KEY WORDS AND PHRASES. $T_{3.5}$-ordered space, $T_{2}$-ordered compactification, defining family of maps, quasi-uniform space, bicompletion.

1991 AMS SUBJECT CLASSIFICATION CODES: 54 F 05, 54 D 35

\section{INTRODUCTION.}

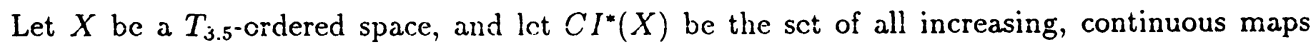
from $X$ into $[0,1]$. A subset $\Phi$ of $C I^{*}(X)$ which induces both the weak order and weak topology on $X$ is called a defining family for $X$. For each such defining family $\Phi$, we construct the smallest $T_{2}$-ordered compactification $K_{\Phi}$ with the property that each member of $\Phi$ can be extended to $K_{\Phi}$. If $\Phi_{1}$ and $\Phi_{2}$ are two defining families for $X$ such that $\Phi_{1} \subseteq \Phi_{2}$, then $K_{\Phi_{1}} \leq K_{\Phi_{2}}$. For each defining family $\Phi$, there is a largest defining family $\hat{\Phi}$ such that $K_{\Phi}=K_{\hat{\Phi}}$. Those defining families which are $\hat{\Phi}$ for some defining family $\Phi$ are called maximal defining families, and if $\Phi$ and $\Psi$ are two maximal defining families, $K_{\Phi} \leq K_{\Psi}$ iff $\Phi=\Psi$. The largest defining family for $X$ is $C I^{*}(X)$, and if $\Phi=C I^{*}(X)$ then $K_{\Phi}$ is the Nachbin (or Stone-Cech ordered) compactification [2].

Each defining family $\Phi$ also generates a quasi-uniformity $\mathcal{V}_{\Phi}$ on $X$ (related to the "usual" quasiuniformity $\mathcal{W}$ on $[0,1])$ which is $T_{0}$ and totally bounded. The bicompletion of $\left(X, \mathcal{V}_{\Phi}\right)$ (as defined in [1]) yields a uniform ordered space which, in turn, gives the compactification $K_{\Phi}$. The maximal defining family $\hat{\Phi}$ is precisely the set of all quasi-uniformly continuous maps from $\left(X, \mathcal{V}_{\Phi}\right)$ into $([0,1], \mathcal{W})$.

\section{PRELIMINARIES.}

If $X$ is a set, we denote by $\mathbf{F}(X)$ the set of all (proper) filters on $X$ and by $\mathbf{U F}(X)$ the set of all ultrafilters on $X$. A non-empty collection $\mathcal{G}$ of subsets of $X$ is called a grill on $X$ if: (1) $\emptyset \notin \mathcal{G}$; (2) $A \in \mathcal{G}$ and $A \subseteq B$ implies $B \in \mathcal{G}$; (3) $A \cup B \in \mathcal{G}$ implies $A \in \mathcal{G}$ or $B \in \mathcal{G}$. With every $\mathcal{F} \in \mathbf{F}(X)$, we associate the grill $\gamma(\mathcal{F})=\{A \subseteq X: X \backslash A \notin \mathcal{F}\}$; equivalently, $\gamma(\mathcal{F})$ is the union of all ultrafilters finer than $\mathcal{F}$.

Let $(X, \leq)$ be a poset; A subset $A \subseteq X$ is increasing (respectively, decreasing) if $x \in A$ and $x \leq y$ (respectively, $y \leq x$ ) implies $y \in A$. If $(X, \leq)$ and $\left(Y, \leq^{*}\right)$ are posets, then a mapping $f$ : 
$(X, \leq) \rightarrow\left(Y, \leq^{*}\right)$ is increasing (respectively, decreasing) if $x \leq y$ implies $f(x) \leq^{*} f(y)$ (respectively, $\left.f(y) \leq^{*} f(x)\right)$.

An ordered space $(X, \tau, \leq)$ consists of a poset $(X, \leq)$ and a topology $\tau$ on $(X, \leq)$ which is convex (meaning that the collection of all $\tau$-open sets which are either increasing or decreasing is a subbase for $\tau)$. Usually an ordered space $(X, \tau, \leq)$ will simply be denoted by $X$. The closed unit interval $[0,1]$ with its usual order and topology is designated by $I$. For any ordered space $X$, let $C I^{*}(X)$ (respectively, $C D^{*}(X)$ ) be the set of all continuous increasing (respectively, decreasing) maps from $X$ into $I$. More generally, for ordered spaces $X$ and $Y, C I(X, Y)$ represents the set of all continuous, increasing functions from $X$ into $Y$.

An ordered space $X$ is said to be $T_{2}$-ordered if the order " $\leq$ " is closed in $X \times X . A T_{2}$-ordered space $X$ which has both the weak order (see Condition $\left(W_{0}\right)$ below) and weak topology induced by $C I^{*}(X)$ is said to be $T_{3.5}$-ordered (or completely regular ordered in the terminology of [2]). Some well-known characterizations of $T_{3.5}$-ordered spaces are summarized in the following proposition.

PROPOSITION 1.1 The following statements about an ordered space $X$ are equivalent.

(1) $X$ is $T_{3.5}$-ordered.

(2) $X$ is a subspace of a compact, $T_{2}$-ordered space.

(3) $X$ satisfies the following conditions:

(i) If $x \in X, A$ is a closed subset of $X$, and $x \notin A$, then there is $f \in C I^{*}(X)$ and

$g \in C D^{*}(X)$ such that $f(x)=g(x)=0$ and $f(y) \vee g(y)=1$, for all $y \in A$;

(ii) If $x \notin y$ in $X$, there is $f \in C I^{*}(X)$ such that $f(y)=0$ and $f(x)=1$.

(4) The order and topology for $X$ are induced by some quasi-uniformity $\mathcal{W}$ on $X$ (i.e., $\cap \mathcal{W}$ is the order for $X$ and the topology of $X$ is the uniform topology of the uniformity $\mathcal{W} \vee \mathcal{W}^{-1}$ ).

Every $T_{3.5}$-ordered space $X$ has a largest $T_{2}$-ordered compactification $\beta_{0} X$ called the Nachbin compactification, which can be constructed by embedding $X$ in the "ordered cube" $I^{C I^{\bullet}(X)}$, with the product order and topology.

Let $X$ be an ordered space. If $\Phi$ is any subset of $C I^{*}(X)$ such that $X$ has the weak order and the weak topology determined by $\Phi$, then $\Phi$ is called a defining family for $X$. More precisely, $\Phi \subseteq C I^{*}(X)$ is a defining family if the following conditions are satisfied:

$\left(W_{\tau}\right)$ For any $\mathcal{F} \in \mathbf{U F}(X), \mathcal{F} \rightarrow x$ in $X$ iff $f(\mathcal{F}) \rightarrow f(x)$ in $I$, for all $f \in \Phi$.

$\left(W_{0}\right)$ For any $(x, y) \in X \times X, x \leq y$ in $X$ iff $f(x) \leq f(y)$ in $I$, for all $f \in \Phi$.

Some rather obvious remarks about defining families are summarized in the next proposition.

PROPOSITION 1.2 Let $X$ be an ordered space.

(1) $X$ is $T_{3.5}$-ordered iff $X$ allows at least one defining family. In particular, $C I^{*}(X)$ is a defining family for every $T_{3.5}$-ordered space.

(2) If $\Phi_{1} \subseteq \Phi_{2} \subseteq C I^{*}(X)$ and $\Phi_{1}$ is a defining family for $X$, then $\Phi_{2}$ is also a defining family for $X$.

2. THE COMPACTIFICATION $K_{\Phi}$.

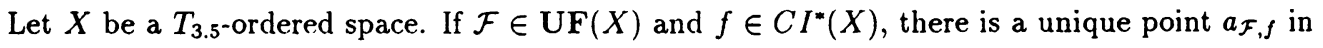
$I$ such that $f(\mathcal{F}) \rightarrow a_{\mathcal{F}, f}$. For any $a \in I$, let $\mathcal{V}(a)$ denote the neighborhood filter at $a$. If $\Phi$ is a defining family for $X$ and $\mathcal{F} \in \mathbf{U F}(X)$, we define the filter $\mathcal{F}_{\Phi}=\vee\left\{f^{-1}\left(\mathcal{V}\left(a_{\mathcal{F}, f}\right)\right): f \in \Phi\right\}$. Note that if $\mathcal{F} \rightarrow x$ in $X$, then $a_{\mathcal{F}, f}=f(x)$ for all $f \in \Phi$, and in this case $\mathcal{F}_{\Phi}$ is simply the neighborhood filter at $x$.

Continuing with the assumptions of the preceding paragraph, let $X_{\Phi}=\left\{\gamma\left(\mathcal{F}_{\Phi}\right): \mathcal{F} \in \mathrm{UF}(X)\right\}$ be the set of grills associated with the filters $\mathcal{F}_{\Phi}$. If $\gamma \in X_{\Phi}$ and $\mathcal{F}, \mathcal{G} \in \mathbf{U F}(X)$ are such that $\mathcal{F} \subseteq \gamma$ and $\mathcal{G} \subseteq \gamma$, then $a_{\mathcal{F}, f}=a_{\mathcal{G}, f}$, for all $f \in \Phi$. It therefore follows that, for each $f \in \Phi$, the function 
$f_{\Phi}: X_{\Phi} \rightarrow I$, defined by $f_{\Phi}(\gamma)=a_{\mathcal{F}, f}$, where $\mathcal{F}$ any ultrafilter that is a subset of $\gamma$, is well-defined. If $i_{\Phi}: X \rightarrow X_{\Phi}$ is defined by $i_{\Phi}(x)=\gamma\left(\dot{x}_{\Phi}\right)$, where $\dot{x}$ is the fixed ultrafilter generated by $\{x\}$, then clearly $i_{\Phi}$ is an injection and the diagram below commutes for every $f \in \Phi$.

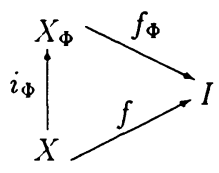

Let $X_{\Phi}$ be equipped with the weak order and weak topology induced by $\left\{f_{\Phi}: f \in \Phi\right\}$. Then $i_{\Phi}$ is an ordered space embedding (i.e., $i_{\Phi}$ is topological embedding, and $x \leq y \Leftrightarrow i_{\Phi}(x) \leq_{\Phi} i_{\Phi}(y)$, where $\leq_{\Phi}$ denotes the order of $X_{\Phi}$ ).

THEOREM 2.1 Let $X$ be a $T_{3.5}$-ordered space and $\Phi$ a defining family for $X$. Then $\left(X_{\Phi}, i_{\Phi}\right)$ is a $T_{2}$-ordered compactification of $X$, and each $f \in \Phi$ has a unique, continuous, increasing extension to $X_{\Phi}$ such that the diagram below commutes.

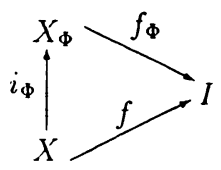

PROOF. The family $\Phi^{\wedge}=\left\{f_{\Phi}: f \in \Phi\right\}$ separates points in $X_{\Phi}$, and therefore $X_{\Phi}$ is $T_{3.5^{-}}$ ordered; in particular, $X_{\Phi}$ is $T_{2}$-ordered. In view of the paragraph preceding the theorem, it remains only to show that $X_{\Phi}$ is compact and $i_{\Phi}(X)$ is dense in $X_{\Phi}$.

Let $\mathcal{A} \in \mathbf{U F}(X)$. For each $\gamma \in X_{\Phi}$, choose an ultrafilter $\mathcal{F}_{\gamma}$ such that $\mathcal{F}_{\gamma} \subseteq \gamma$; in particular, if $\gamma=\gamma\left(\mathcal{F}_{\Phi}\right)$ where $\mathcal{F} \rightarrow x$ in $X$, define $\mathcal{F}_{\gamma}=\dot{x}$. If $B \subseteq X$, let $B^{*}=\left\{\gamma \in X_{\Phi}: B \in \mathcal{F}_{\gamma}\right\}$. Then, define $\mathcal{F}_{\mathcal{A}}=\left\{A \subseteq X: A^{*} \in \mathcal{A}\right\}$; one easily verifies that $\mathcal{F}_{\mathcal{A}}$ is an ultrafilter. We shall show that $\mathcal{A} \rightarrow \gamma\left(\mathcal{F}_{\mathcal{A}}\right)$ in $X_{\Phi}$. For this purpose, it suffices to show that $f_{\Phi}(\mathcal{A}) \rightarrow f_{\Phi}\left(\gamma\left(\mathcal{F}_{\mathcal{A}}\right)\right)=a_{\mathcal{F}_{A}, f}$. for all $f \in \Phi$. Given $f \in \Phi$, let $U$ be a closed neighborhood of $a_{\mathcal{F}_{A}, f}$ in $I$. We first observe that $f\left(\mathcal{F}_{\mathcal{A}}\right) \rightarrow a_{\mathcal{F}_{A}, f}$, and hence $f^{-1}(U) \in \mathcal{F}_{\mathcal{A}}$, which implies $\left(f^{-1}(U)\right)^{*} \in \mathcal{A}$. Then note that $f\left(\mathcal{F}_{\mathcal{A}}\right) \rightarrow a_{\mathcal{F}_{A}, f}$; consequently $f_{\Phi}^{-1}(U) \in \mathcal{A}$, and $f_{\Phi}(\mathcal{A}) \rightarrow a_{\mathcal{F}_{A}, f}$. Thus $X_{\Phi}$ is compact.

Finally, let $\gamma \in X_{\Phi}$ and, for $B \subseteq X$, let $B^{*}$ be defined as in the preceding paragraph. If $\mathcal{F} \in \mathbf{U F}(X)$ and $\mathcal{F} \subseteq \gamma$, let $\mathcal{F}^{*}$ be the filter on $X_{\Phi}$ generated by $\left\{F^{*}: F \in \mathcal{F}\right\}$. One easily shows that $\mathcal{F}^{*} \rightarrow \gamma$ in $X_{\Phi}$. Since $i_{\Phi}(\mathcal{F}) \geq \mathcal{F}^{*}$, it follows that $i_{\Phi}(X)$ is dense in $X_{\Phi}$. I

The compactification $\left(X_{\Phi}, i_{\Phi}\right)$ of $X$ determined by a defining family $\Phi$ will be denoted by $K_{\Phi}$. By the preceding theorem, each $f \in \Phi$ has a unique extension $f_{\Phi} \in C I^{*}\left(X_{\Phi}\right)$. If $Y$ is any compact, $T_{2}$-ordered space, we define $C I_{\Phi}(X, Y)=\left\{f \in C I(X, Y): h \circ f \in \Phi\right.$, for all $\left.h \in C I^{*}(Y)\right\}$. The next theorem establishes that each $f \in C I_{\Phi}(X, Y)$ can be "lifted" relative to $K_{\Phi}$.

THEOREM 2.2 Let $X$ be a $T_{3.5}$-ordered space, $\Phi$ a defining family for $X$, and $Y$ a compact, $T_{2}$-ordered space. If $g \in C I_{\Phi}(X, Y)$, then there is a unique $g_{\phi} \in C I\left(X_{\Phi}, Y\right)$ such that the diagram below commutes.

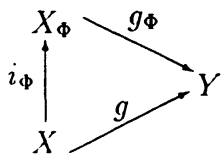

PROOF. Let $g \in C I_{\Phi}(X, Y)$ and $\gamma \in X_{\Phi}$; assume $\mathcal{F}$ is an ultrafilter and $\mathcal{F} \subseteq \gamma$. Define $g_{\Phi}: X_{\Phi} \rightarrow Y$ as following: $g_{\phi}(\gamma)=y_{F, g}$, where $y_{\mathcal{F}, g}$ is the unique limit of $g(\mathcal{F})$ in $Y$. Using the facts that $g \in C I_{\Phi}(X, Y)$ and $C I^{*}(Y)$ separates points in $Y$, we see that $g_{\phi}(\gamma)$ is independent of the ultrafilter $\mathcal{F}$ which represents $\gamma$, so $g_{\phi}$ is well defined. 
If $h \in C I^{*}(Y)$, let $h^{\prime}=h \circ g$. Then we observe that the preceding definition of $g$ makes the following diagram commutes:

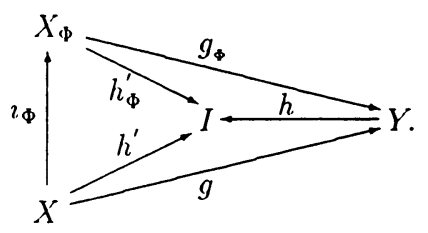

If $\gamma \leq \delta$ in $X_{\Phi}$, then $h_{\Phi}^{\prime}(\gamma) \leq h_{\Phi}^{\prime}(\delta), \forall h \in C I^{*}(Y)$, which implies $h\left(g_{\phi}(\gamma)\right) \leq h\left(g_{\phi}(\delta)\right)$ holds for all $h \in C I^{*}(Y)$. Since $Y$ has the weak order induced by $C I^{*}(Y), g_{\phi}(\gamma) \leq g_{\phi}(\delta)$. Thus $g_{\phi}$ is increasing. A similar argument, based on $Y$ having the weak topology induced by $C I^{*}(Y)$, shows that $g_{\phi}$ is continuous. The uniqueness of $g_{\phi}$ is obvious because all spaces involved are Hausdorff. I

We omit the simple proof of the next proposition.

PROPOSITION 2.3 If $\Phi$ is a defining family for a $T_{3.5}$-ordered space $X$, then $\Phi^{\prime}=\left\{f_{\Phi}: f \in\right.$ $\left.C I^{*}(X)\right\}$ is a defining family for $X_{\Phi}$.

Starting with a $T_{3.5}$-ordered space $X$ and a defining family $\Phi$ for $X$, it follows that $\Phi^{\prime}$ and $C I^{*}\left(X_{\Phi}\right)$ are both defining families for $X_{\Phi}$, and it is clear that $\Phi^{\prime} \subseteq C I^{*}\left(X_{\Phi}\right)$. Let $\hat{\Phi}=\{f \in$ $C I^{*}(X)$ : there is $g \in C I^{*}\left(X_{\Phi}\right)$ such that $\left.f=g \circ i_{\Phi}\right\}$; in other words, $\hat{\Phi}$ consists of all members of $C I^{*}(X)$ which have a continuous, increasing extension in $C I^{*}\left(X_{\Phi}\right)$. Clearly $\Phi \subseteq \hat{\Phi}$, and so $\hat{\Phi}$ is a defining family for $X$. Note that $(\hat{\Phi})^{\prime}=C I^{*}\left(X_{\Phi}\right)$, and since $(\hat{\Phi})^{\prime}$ is, by Proposition 2.3 , a defining family for $X_{\hat{\phi}}$, it follows that $X_{\hat{\Phi}}=X_{\Phi}$. These observations yield the following result.

PROPOSITION 2.4 If $\Phi$ is a defining family for a $T_{3.5}$-ordered space $X$, then $\hat{\boldsymbol{\Phi}}=\{f \in$ $C I^{*}(X)$ : there is $g \in C I^{*}\left(X_{\Phi}\right)$ such that $\left.f=g \circ i_{\Phi}\right\}$ is the largest defining family for $X$ such that $K_{\Phi}=K_{\hat{\Phi}}$.

THEOREM 2.5 Let $\Phi, \Psi$ be defining families for a $T_{3.5}$-ordered space.

(a) If $\Phi \subseteq \Psi$, then $K_{\Phi} \leq K_{\Psi}$.

(b) $K_{\Phi} \leq K_{\Psi}$ iff $\hat{\Phi} \subseteq \hat{\Psi}$.

PROOF. (a) $\Phi \subseteq \Psi$ implies $\hat{\Phi} \subseteq \hat{\Psi}$. Considering the diagram

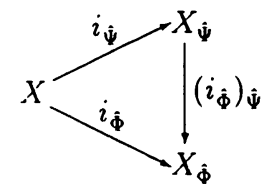

and applying Theorem 2.2, we see that $\left(i_{\hat{\phi}}\right)_{\hat{\psi}}$ is increasing and continous. Thus $K_{\Phi}=K_{\hat{\Phi}} \leq K_{\hat{\Psi}}=$ $K_{\Psi}$.

(b) If $K_{\Phi} \leq K_{\Psi}$, then there is an increasing, continous map $\sigma$ making the diagram

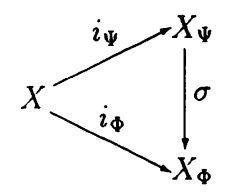

commute. Each member of $\hat{\Phi}$ has the form $f \circ i_{\Phi}$ for some $f \in C I^{*}\left(X_{\Phi}\right)$. But $f \circ i_{\Phi}=f \circ \sigma \circ i_{\Psi}$ is also in $\hat{\Psi}$. Thus $\hat{\Phi} \subseteq \hat{\Psi}$. The converse follows from (a). I

If $X$ is a $T_{3.5}$-ordered space, let $\mathrm{DF}(X)$ be the poset of all defining families, ordered by inclusion. Two defining families $\Phi$ and $\Psi$ in $\mathrm{DF}(X)$ are equivalent if $K_{\Phi}=K_{\Psi}$ (i.e., if $K_{\Phi}$ and $K_{\Psi}$ are equivalent compactifications of $X$ in the usual sense). Thus $\operatorname{DF}(X)$ is partitioned into equivalent 
classes, and each equivalent class $\langle\Phi\rangle$ contains a largest member $\hat{\Phi}$ which we call a maximal defining family.

COROLLARY 2.6 Let $X$ be a $T_{3.5}$-ordered space, $K=\left(X^{\prime}, \phi\right)$ a $T_{2}$-ordered compactification of $X$, and $\Phi \in \mathbf{D F}(X)$ such that each $f \in \Phi$ has an extension $f^{\prime} \in C I^{*}\left(X^{\prime}\right)$. Then $K_{\Phi} \leq K$.

COROLLARY 2.7 For a $T_{3.5}$-ordered space, the correspondence $\Phi \longleftrightarrow K_{\Phi}$ is bijective and order-preserving between the maximal defining families for $X$ and the $T_{2}$-ordered compactifications of $X$.

\section{DEFINING FAMILIES AND QUASI-UNIFORMITIES.}

This concluding section is based on the results of Fletcher and Lindgren [1], and to some extent we borrow their notation.

Let $(X, \mathcal{V})$ be a quasi-uniform space; the associated uniformity $\mathcal{V} \vee \mathcal{V}^{-1}$ will be denoted by $\mathcal{V}^{*}$. Recall that $(X, \mathcal{V})$ is $T_{0}$ iff $\cap \mathcal{V}$ is a partial order (or, equivalently, $\left(X, \mathcal{V}^{*}\right)$ is $\left.T_{2}\right)$, and totally bounded iff, for each $U \in \mathcal{V}$, there is a finite cover $\left\{A_{1}, \cdots, A_{n}\right\}$ of $X$ such that $A_{\imath} \times A_{\imath} \subseteq U$, for $i=1, \cdots, n$. Note that $\mathcal{V}$ is totally bounded iff $\mathcal{V}^{*}$ is totally bounded.

Every $T_{0}$, quasi-uniform space $(X, \mathcal{V})$ induces a uniform ordered space $(X, \mathcal{U}, \leq)$, where $\mathcal{U}=\mathcal{V}^{*}$ and " $\leq$ " $=\cap \mathcal{V}$; also associated with $(X, \mathcal{V})$ is the $T_{3.5}$-ordered space $(X, \tau, \leq)$, where $\tau=\tau_{\mathcal{V}}$. and " $\leq$ " is again $\cap \mathcal{V}$. Furthermore, for every compact, $T_{2}$-ordered space $(X, \tau, \leq)$, there is a unique quasi-uniformity $\mathcal{V}$ on $X$ such that $\tau=\tau_{\mathcal{V}}$ and " $\leq "=\cap \mathcal{V}$ (Theorem 4.21, [1]). In particular, for the compact, $T_{2}$-ordered space $I$, the unique compatible quasi-uniformity, denoted here by $\mathcal{W}$, has a base of sets of the form $W_{\iota}=\{(x, y) \in I \times I: x-y \leq \epsilon\}$, where $\epsilon>0$.

For a quasi-uniform space $(X, \mathcal{V})$, let $Q U C(X, \mathcal{V})$ be the set of all quasi-uniformly continous maps from $(X, \mathcal{V})$ into $(I, \mathcal{W})$. If $X=(X, \tau, \leq)$ is the $T_{3.5}$-ordered space associated with $(X, \mathcal{V})$, it is clear that $Q U C(X, \mathcal{V}) \subseteq C I^{*}(X)$. It is shown in Theorems 3.29 and 3.33 of [1] that every $T_{0}$, quasiuniform space $(X, \mathcal{V})$ has a bicompletion $((\tilde{X}, \tilde{\mathcal{V}}), \jmath)$ such that $\left(\left(\tilde{X},(\tilde{\mathcal{V}})^{*}\right), \jmath\right)$ is the unique uniform space completion of $\left(X, \mathcal{V}^{*}\right)$, and each $f \in Q U C(X, \mathcal{V})$ has a unique extension in $Q U C(\tilde{X}, \tilde{\mathcal{V}})$. These observations lead to the following proposition.

PROPOSITION 3.1 Let $(X, \mathcal{V})$ be a $T_{0}$, totally bounded quasi-uniform space with associated $T_{3.5}$-ordered space $(X, \tau, \leq)$, and let $((\tilde{X}, \tilde{\mathcal{V}}), \jmath)$ be the bicompletion of $(X, \mathcal{V})$. If $(\tilde{X}, \tilde{\tau}, \tilde{\leq})$ is the $T_{3.5}$-ordered space associated with $(\tilde{X}, \tilde{\mathcal{V}})$, then $\tilde{K}=((\tilde{X}, \tilde{\tau}, \tilde{\leq}), \jmath)$ is a $T_{2}$-ordered compactification of $(X, \tau, \leq)$.

THEOREM 3.2 Let $X$ be a $T_{3.5}$-ordered space and $\Phi \in \mathbf{D F}(X)$. Let $\mathcal{V}_{\Phi}$ be the weak uniformity on $X$ induced by $\Phi$ relative to $(I, \mathcal{W})$. Let $\left(\left(\tilde{X}_{\Phi}, \tilde{\mathcal{V}}_{\Phi}\right), \jmath\right)$ be the bicompletion of $\left(X, \mathcal{V}_{\Phi}\right)$, and $\tilde{K}_{\Phi}=\left(\left(\tilde{X}_{\Phi}, \tilde{\tau}_{\Phi}, \tilde{\leq}\right), \jmath\right)$ be the $T_{2}$-ordered compactification of $X$ induced by the bicompletion. Then $\tilde{K}_{\Phi}=K_{\Phi}$.

PROOF. Let $\mathcal{V}$ be the unique, $T_{0}$ totally bounded quasi-uniformity on $X_{\Phi}$ whose associated $T_{3.5}$-ordered space is the compactification $\left(\left(X_{\Phi}, \tau_{\Phi}, \leq_{\Phi}\right), i_{\Phi}\right)$ derived from $\Phi$. The latter space has the weak order and topology induced by $\Phi^{\prime}$ (see Proposition 2.3) relative to $I$, and hence $\mathcal{V}$ is the weak quasi-uniformity on $X_{\Phi}$ induced by $\Phi^{\prime}$ relative to $(I, \mathcal{W})$. If $\mathcal{U}=\left(i_{\Phi}\right)^{-1}(\mathcal{V})$ is the restriction of $\mathcal{V}$ to $X$, then $\mathcal{U}$ is the weak quasi-uniformity on $X$ induced by $\Phi$ relative to $(I, \mathcal{W})$. In other words, $\mathcal{U}=\mathcal{V}_{\Phi}$. Since the $T_{2}$-ordered compartification associated with a $T_{0}$, totally bounded quasiuniformity is unique (up to equivalence), $\tilde{K}_{\Phi}=K_{\Phi}$. I

COROLLARY 3.3 Let $X$ be a $T_{3.5}$-ordered space and $\Phi \in \mathrm{DF}(X)$. Then $\hat{\phi}=Q U C\left(X, \mathcal{V}_{\Phi}\right)$.

PROOF. By Theorem 3.29, [1], earh $f \in Q U C\left(X, \mathcal{V}_{\Phi}\right)$ can be extended to the compactification $\tilde{K}_{\Phi}=K_{\Phi}$; thus $Q U C\left(X, \mathcal{V}_{\Phi}\right) \subseteq \hat{\Phi}$. ('onversely, each $f \in \hat{\Phi}$ has a unique, increasing, continuous extension to $K_{\Phi}=\tilde{K}_{\Phi}$, and this extension of $f$ is quasi-uniformly continuous from $\left(\tilde{X}_{\Phi}, \tilde{\mathcal{V}}_{\Phi}\right)$ into 
$(I, \mathcal{W})$. Thus $f \in Q U C\left(X, \mathcal{V}_{\Phi}\right)$. I

COROLLARY 3.4 Let $(X, \mathcal{V})$ be a $T_{0}$, totally bounded quasi-uniform space with associated compact, $T_{2}$-ordered space $X=(X, \tau, \leq)$. Then $\Phi=Q U C(X, \mathcal{V})$ is a maximal defining family for $X$ and $\mathcal{V}=\mathcal{V}_{\Phi}$.

COROLLARY 3.5 Let $X$ be a $T_{3.5}$-ordered space. Then $\mathcal{V} \longleftrightarrow Q U C(X, \mathcal{V})$ is bijective and order-preserving between the $T_{0}$, totally bounded quasi-uniformities which induce $X$ and the maximal defining families for $X$.

\section{REFERENCES}

[1 ] P. Fletcher and W. Lindgren, Quası-Uniform Spaces, Lecture Notes in Pure and Applied Mathematics, Vol. 77, Marcel Dekker, New York (1982).

[2 ] L. Nachbin, Topology and Order, Van Nostrand Math. Studies No. 4, Princeton (1965). 


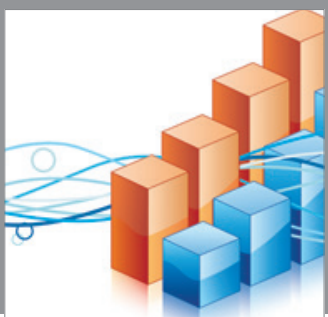

Advances in

Operations Research

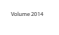

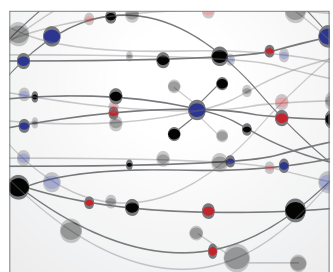

\section{The Scientific} World Journal
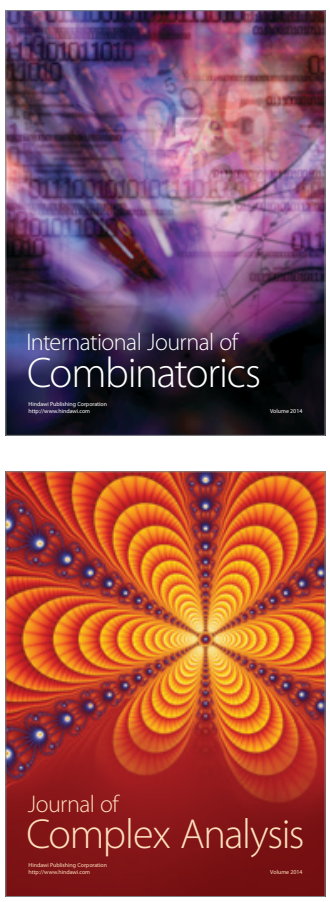

International Journal of

Mathematics and

Mathematical

Sciences
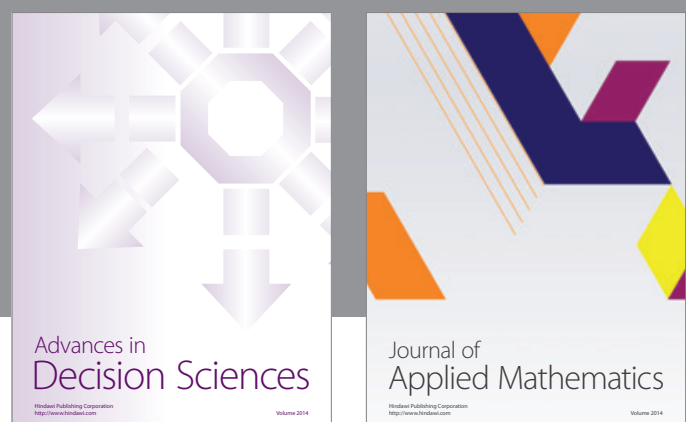

Journal of

Applied Mathematics
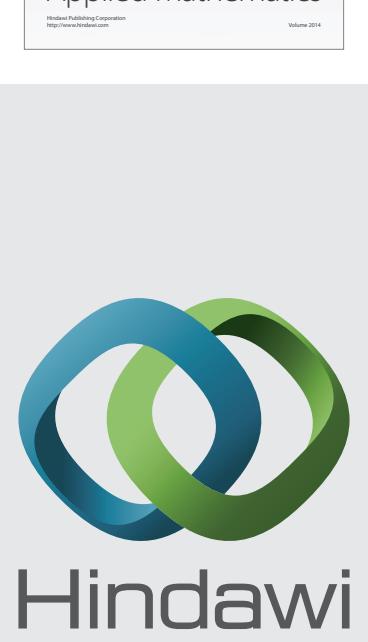

Submit your manuscripts at http://www.hindawi.com
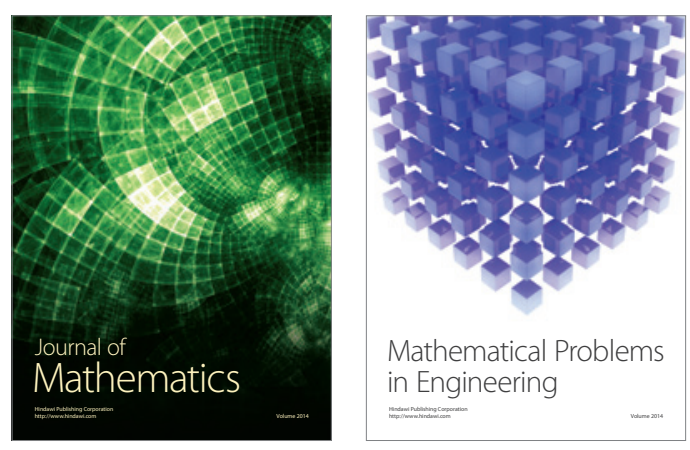

Mathematical Problems in Engineering
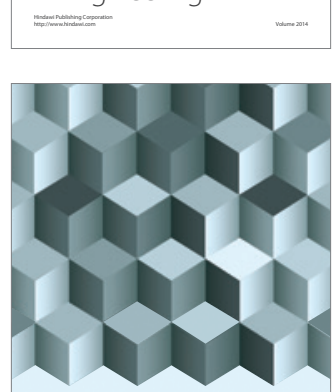

Journal of

Function Spaces
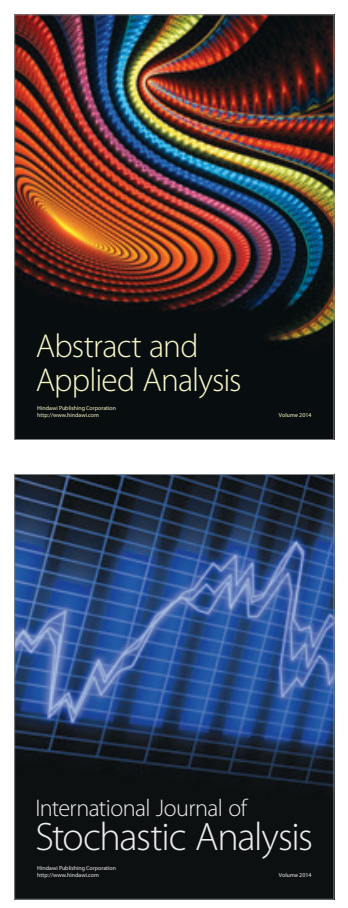

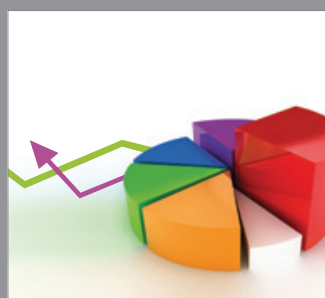

ournal of

Probability and Statistics

Promensencen
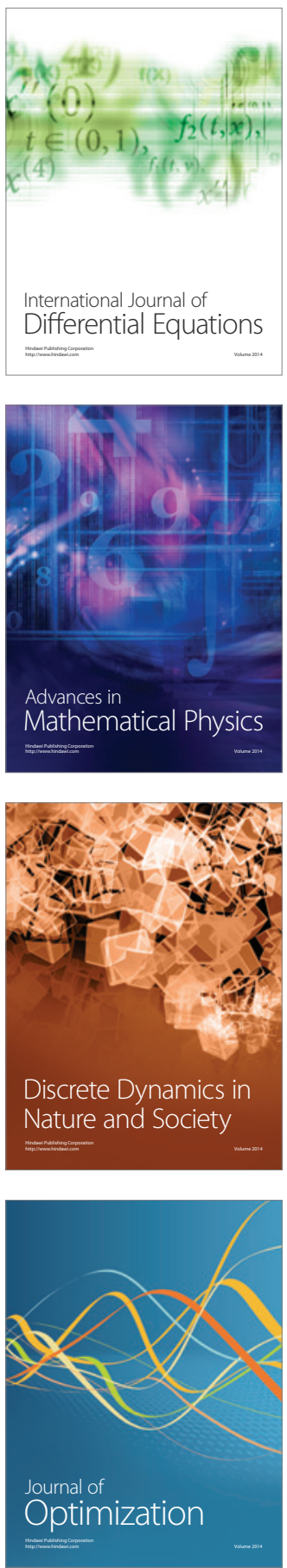